\title{
Wie viele haben Anti-JC-Virus-Antikörper?
}

Fragestellung: Wie hoch ist die Prävalenz der Antikörper gegen das JC-Virus unter deutschen Patienten mit Multipler Sklerose (MS), die mit Natalizumab therapiert werden und wie hoch ist die (jährliche) Konversionsrate?

Hintergrund: Die progressive multifokale Leukenzephalopathie (PML) ist eine sehr seltene Erkrankung, die fast ausschließlich bei immunsupprimierten Patienten mit HIV, Zustand nach Knochenmarktransplantation (KMT) oder hämatologischen Grunderkrankungen auftritt. Unter der Therapie mit Natalizumab sind weltweit bereits etwa 270 PML-Fälle (Stand September 2012) bei MS-Patienten aufgetreten, wobei die Letalität mit etwa $20-25 \%$ zwar immer noch hoch, aber deutlich niedriger als bei Patienten mit HIV oder nach KMT ist (jeweils > 80\%).

Das PML-Risiko bei MS-Patienten steigt mit der Dauer der Natalizumab-Behandlung (länger als 24 Monate), immunsuppressiven beziehungsweise chemotherapeutischen Vorbehandlungen sowie dem Nachweis einer bereits stattgefundenen Exposition zum JC-Virus. Inzwischen steht ein Antikörpertest zur Verfügung, der es erlaubt, Patienten auf Antikörper gegen das JC-Virus zu testen. Da sich bisher keine klaren Expositionsmuster erkennen lassen und die Seroprävalenz mit dem Alter zuzu-

Trampe AK, Hemmelmann C, Stroet A et al. Anti-JC virus antibodies in a large German natalizumab-treated multiple sclerosis cohort. Neurology 2012; 78 : $1736-42$ nehmen scheint, bleibt die Frage, wie lange ein Antikörpernegativer Patient tatsächlich auch negativ bleibt und mit welcher Konversionsrate unter der Behandlung mit Natalizumab grundsätzlich gerechnet werden muss.
Patienten und Methodik: Für diese Studie standen 2.782 Blutproben von 2.253 MS-Patienten aus Deutschland zur Verfügung, die zwischen 2007 und 2010 mit Natalizumab behandelt worden waren. Von circa $10 \%$ der Patienten lagen mehrere Proben vor, sodass diese im Hinblick auf die Konversionsrate untersucht werden konnten. Darüber hinaus lagen von zehn PMLFällen Serumproben vor, die vor Auftreten der Erkrankung abgenommen waren.

Ergebnisse: Auch in dieser Studie zeigt sich eine JCV-Antikörperprävalenz von durchschnittlich $58,8 \%$, die bei Männern etwas höher war als bei Frauen und mit dem Alter anstieg. In der longitudinalen Untersuchung der Patienten konvertierten 19 von $194(=9,8 \%)$ innerhalb von durchschnittlich 7,7 Monaten von Serum-negativ zu JC-Virus-Antikörper-positiv.

Auf der anderen Seite revertierten 4,7\% der untersuchten Patienten von JC-Virus-Antikörper-positiv zu negativ über einen durchschnittlichen Zeitraum von 7,9 Monaten. Die von zehn an PML-erkrankten Patienten vor der Diagnose entnommenen Proben waren alle positiv. Interessanterweise war der Antikörpertiter bei diesen Patienten am höchsten.

Schlussfolgerungen: Die Prävalenz der JCV-Antikörper entspricht mit circa $58 \%$ auch hier der Rate, die auch in der anderen deutschen Studie ermittelt beziehungsweise aus anderen Ländern (USA, Italien, Schweden) berichtet worden ist. Die Konversionsrate war in dieser Studie mit 9,8\% deutlich höher, als die bisherigen Berechnungen, die von Konversionsraten von rund 2-3\% im Jahr ausgingen. Neu ist der Aspekt, dass Patienten offensichtlich auch revertieren können.

\section{-Kommentar von Prof. Volker Limmroth}

\section{Jährlicher Test auf JCV-Antikörper für Antikörper-negative Patienten}

Die Beobachtung, dass die Konversionsrate in einigen Kohorten offensichtlich knapp $10 \%$ betragen kann, ist wichtig und bestätigt die derzeit gängige Praxis, JCV-Antikörper-negative Patienten jährlich neu zu testen. Streng genommen könnte in dieser Kohorte die jährliche Konversionsrate sogar noch höher gewesen sein, denn diese bezog sich auf einen gemittelten Untersuchungszeitraum von etwas über sieben Monaten. Die Tatsache, dass alle PML-Fälle, von denen Serum vor der Erkrankung vorhanden waren, auch positiv waren, bestätigt ebenfalls den Wert des Antikörpertests. Die Beobachtung, dass wiederum bis zu $5 \%$ der Antikörper-positiven Patienten revertieren, ist schwer zu interpretieren. Ob hier ein Testproblem im Sinne eines falsch-negativen Ergebnisses vorliegt oder die Antikörperproduktion tatsächlich nach einem bestimmten Zeitraum wieder abnimmt, ist nicht klar und muss weiter untersucht werden. Interessant ist auch die Beobachtung, dass die Antikörpertiter bei den PML-Patienten am höchsten waren. Könnte hier durch zukünftige Untersuchungen eine Art „Grenz-
Titer" ermittelt werden, würde der Test im Hinblick auf die Risikostratifizierung weiter an Bedeutung gewinnen. Wichtigste Botschaft der Studie ist jedoch: AK-negative Patienten sollten unter einer Natalizumab-Therapie jährlich auf JCV-Antikörper getestet werden.

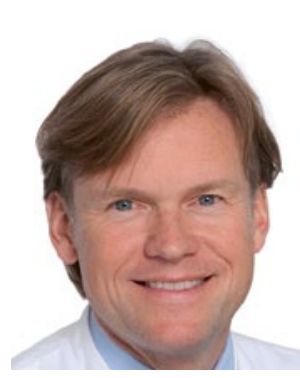

Prof. Dr. med. Volker Limmroth, Köln

Chefarzt der Klinik für Neurologie und Palliativmedizin Köln-Merheim E-Mail: LimmrothV@kliniken-koeln.de 